\title{
A New-Generation Method for Quick and Owren PT
}

\author{
Juha Horsti, ${ }^{1, *}$ Helena Uppa ${ }^{1}$ and Juhani A. Vilpo ${ }^{2}$
}

${ }^{I}$ Tampere University Hospital, Centre for Laboratory Medicine, P.O. Box 2000, FIN-33521 Tampere, Finland; Univer-
sity of Tampere, FIN-33521 Tampere, Finland. ${ }^{2}$ Department of Clinical Chemistry, Centre for Laboratory Medicine,
Tampere University Hospital and University of Tampere.

\begin{abstract}
Oral anticoagulant therapy (OAT) calls for continuous control by prothrombin time (PT) test, as the therapeutic range in INR units is very narrow. Warfarin (or coumarin) inhibits coagulation factor synthesis in the liver, but at the same time inactive coagulation factors are formed. The aim here was to measure "active coagulation factors" and inhibition in calibrator kits and patient plasmas by a new method for Quick and Owren PT.

Four calibration kits and 200 plasma samples obtained from OAT patients were assessed using Quick and Owren PT for $\mathrm{INR}_{\text {Tot }}$ (active coagulation factors + inhibition) and $\mathrm{INR}_{\mathrm{Acf}}$ (only active coagulation factors). Conspicuous variation in inhibition was noted between the four calibration kits. The new- generation PT method develops anticoagulation therapy based on active coagulation factors in vivo and improves INR result harmonization for Quick and Owren PT reagents. This new approach improves Quick PT reliability.
\end{abstract}

Key Words: PT, prothrombin time, oral anticoagulant therapy.

\section{INTRODUCTION}

The prothrombin time (PT) test is used mainly to control oral anticoagulant therapy (OAT). PT is the most widely used coagulation test in clinical laboratories and in year 2000 about 800 million tests are carried out globally. The number of tests is increasing about $10 \%$ a year. OAT is based on the ability of warfarin as a vitamin $\mathrm{K}$ antagonist, to slow down the synthesis of active coagulation factors in the liver (F II, F VII, F IX and F X). Warfarin medication requires continuous monitoring to prevent the serious consequences of thrombosis or bleeding, which may mean patient death [1]. Mortality has been seen to be strongly related to the level of the International Normalized Ratio (INR), and accuracy in patient care is very important.

The prothrombin time is commonly measured by either the "Quick PT", which is based on the technique described by Quick and co-workers in $1935[2,3]$ or by the "Owren PT" [4] (combined thromboplastin reagent). The latter is the predominant approach used in the Nordic countries, Benelux, and Japan, both methods being nevertheless suitable for the control of anticoagulant treatment. The WHO recommendation for the use of INR aims to harmonize PT results for OAT regardless of the reagent, instrument or method used $[5,6]$.

Though expectations of harmonization of results using INR are not fulfilled in routine measurements [7-13], the therapeutic ranges are globally the same as those of INR for different clinical indications.

*Address correspondence to this author at the Tampere University Hospital, Centre for Laboratory Medicine, P.O. Box 2000, FIN-33521 Tampere, Finland; Tel: +358 3331 75290; Fax: +358 3 75554;

E-mail: juha.horsti@pshp.fi
The editorials of Clinical Chemistry have posed the critical question and sought answers: "Has the Time Arrived to Replace the Quick Prothrombin Time Test for Monitoring Oral Anticoagulant Therapy ?"[7]. Horsti compared the Quick and Owren PT methods for harmonization of INR results and concluded that Quick PT yields clinically divergent and Owren PT clinically acceptable INR results [12]. Lindahl and colleaques noted that the current International Sensitivity Index (ISI) calibration standardization procedure is complex and recommend normal plasma calibration with dilutions [14]. Horsti and associates in a recent study presented "A new-generation prothrombin time method", which measures active coagulation factors F II, F VII, F X and separately the inhibition caused by inactive coagulation factors F II, F VII, F X , which totally or partly lack gamma carboxyglutamic acid for factor activation [15-18]. This study by Owren PT found that OAT patients should be medicated using INR from active coagulation factors and calibrators should not contain inhibitors.

The aim of this present study was to establish whether "The New-generation PT method" is appropriate for Quick PT and whether we can harmonize INR results between Owren and Quick PT's, and to what extent ISI calibrators contain inactive coagulation factors for Quick PT.

\section{MATERIALS AND METHODOLOGY}

\section{Patients and Blood Sampling}

Venous blood samples were obtained from 10 normal subjects and 210 hospital and health-centre patients for whom the PT time test was requested for the monitoring of oral anticoagulant therapy. In our region a "P-INR" test code is used for this purpose. The patient samples thus represented all possible phases of anticoagulation: (i) pre-treatment, (ii) 
dose-adjusting phase, and (iii) steady- state phase. All procedures were approved by our institution's responsible committee in accordance with the Helsinki Declaration of 1975. Blood $(1.8 \mathrm{~mL})$ was drawn into citrate coagulation tubes (Greiner Labortechnik GmbH, Vacuette cat. no. 454322, 9NC) containing $0.2 \mathrm{~mL} 0.109 \mathrm{~mol} / \mathrm{L}(3.2 \%)$ citrate solution. The sample needle (Terumo, Venoject needle, Quick Fit, cat. no. MN-2138MQ) was 0.8 x $40 \mathrm{~mm}$. Sample tubes were centrifuged at $1850 \mathrm{~g}$ for $10 \mathrm{~min}$ at $20{ }^{\circ} \mathrm{C}$ to separate plasma. All measurements were commenced within 8 hours of blood collection.

\section{PT Determination}

The PT coagulation times were measured using a fully automated BCS coagulation analyser (DadeBehring Coagulation System, DadeBehring, Marburg, Germany).

For the one-stage propthrombin time with Quick PT, 100 $\mu \mathrm{L}$ of coagulation reagent was added to $50 \mu \mathrm{L}$ of citrated plasma and for dilution the sample volumes were $100 \mu \mathrm{L}$ $+25 \mu \mathrm{L}+25 \mu \mathrm{L}$ (a physiologic salt solution, Natriumklorid 9 $\mathrm{mg} / \mathrm{mL}, 500 \mathrm{ml}$ from Kabi). The reagent was Dade Innovin cat. no. B4212-50 (recombinant human tissue thromboplastin, DadeBehring Marburg GmbH), lot 536928, ISI for BCS 0.92 .

Owren PT method (combined thromboplastin reagent): the coagulation reactionmixture contained $10 \mu \mathrm{L}$ of citrated sample plasma, $60 \mu \mathrm{L}$ of diluent and $140 \mu \mathrm{L}$ of reagent for normal PT measurement and $5 \mu 1$ of citrated sample plasma, $65 \mu \mathrm{L}$ of diluent and $140 \mu \mathrm{L}$ of reagent for patient and calibrator measurements using the new PT method.

The reagent was Nycotest PT, cat. no. 1002488 (rabbit brain thromboplastin) and a diluent (Nycotest PT, dilution liquid, cat.no. 1002485) from Axis-Shield as, lot 10112954, ISI $=1.07$.

\section{ISI Calibration}

Two local ISI calibrator kits were used: (i) "Svensk nationell kalibrator för protrombinkomplexaktiviet", from Equalis, lot 11, 12, Cal 1=0.85 INR and Cal 2=3.19 INR (used mainly in Sweden and Norway). (ii) "ISIkalibraattorikitti", cat.no. B10000150, from Bioclin, lot 8, Cal 1=2.07 INR, Cal 2=3.52 INR and Cal 3=1.0 INR (used mainly in Finland).

Further, two commercial ("manufacturer calibration") ISI calibration kits were used: (i) Etaloquick cat. no.00496 from Diagnostica Stago lot 041555. Cal $1=0.91$ INR, Cal $2=3.24$ INR and Cal $3=4.90$ INR, and. (ii) PT-Multi Calibrator cat.no. OPAT 035 from DadeBehring, lot 35422. Cal 1=1.01 INR, Cal 2=1.30 INR, Cal 3=1.65 INR, Cal 4=2.97 INR, Cal $5=4.00 \mathrm{INR}, \mathrm{Cal} 6=5.29 \mathrm{INR}$.

\section{Determination of Minimal PT Time and Respective INR}

The construction of a PT sec (y axis) versus C (where C is the dilution factor of normal plasma, OAT plasma, or calibrator) plot shows, at the intercept of the line obtained from the experiment, the so-called minimal clotting time $\left(\mathrm{t}_{\min }\right)$. The inhibition effect can be calculated from the differences in intercepts of the unknown sample and normal plasma (or INR "zero" calibrator). In practice, only two dilutions are required for each determination [15].
The inhibition principle on the y axis is illustrated in Fig. $\mathbf{1} \mathbf{A}$ and $\mathbf{B}$ [15]. We further calculated the difference in intercepts (= inhibition) in INR units and subtracted this from total $\mathrm{INR}_{\mathrm{Tot}}$.

$$
\mathrm{INR}_{\text {Acf }}=\mathrm{INR}_{\text {Tot }}-\mathrm{INR}_{\text {Inh }}
$$

INRs were calculated using the formula: INR $=($ sample $\mathrm{sec} /$ normal $\left._{\mathrm{sec}}\right)^{\mathrm{ISI}}$

Patent pending for method (EP 1861720, WO 2006100346).

\section{Analytical Imprecision and Statistics}

The within-run precision of PT tests was measured using one patient plasma sample ( $\mathrm{n}=10$ determinations) with an INR value in the therapeutic range, i.e., approx. 2.2 INR. The respective CVs were: $2.6 \%$ for Dade Innovin and $1.6 \%$ for Nycotest PT. This is consistent with our previous observations with a broader spectrum of reagents [13]. The Microsoft Excel 5.0 program was used to obtain the correlation functions and INR results.

\section{RESULTS}

Inhibition in INR units and percentages using the Quick and Owren PT on four commercial calibrator kits is presented in Table $\mathbf{1}$ and Fig. (1).

The inhibition is dependent on thromboplastin sensitivity and the PT method used. The extent of inhibition varies markedly between calibrator kits and creates disharmony in calibration and thus in OAT patient INR. The corrected new method gives the true $\mathrm{INR}_{\text {Acf }}$ value of active coagulation factors in the calibrator.

Inhibition was demonstrable using Owren and Quick PT in all calibrators with INR values greater than 1. As expected, the inhibition increased concomitantly with the increase in calibrator INRs.

The local Nordic calibrators Bioclin and Equalis behave similarly to Quick $(1.0 ; 1.95 ; 2.72 ; 0.85 ; 2.91)$ and $(1.0 ; 1.75$; $2.78 ; 0.85 ; 2.88$ ) Owren PT in respect of active coagulation factors. Manufacturer's calibrators show more difference between the Quick $(1.01 ; 1.17 ; 1.39 ; 2.23 ; 3.00 ; 3.61 ; 1.00$ $2.22 ; 3.09)$ and the Owren PT $(1.01 ; 1.26 ; 1.55 ; 2.64 ; 3.65$; $4.34 ; 1.00 ; 2.54 ; 3.25)$ methods with regard to active coagulation factors. The plasma material in the calibrators is separate from patient samples, as they contain preservatives and additives.

Individual 200 OAT patient samples were measured by the Quick and Owren PT in increasing order for $\mathrm{INR}_{\mathrm{Tot}}$ and $\mathrm{INR}_{\text {Acf }}$ (Fig. 2). Higher levels of anticoagulant medication reduce the amount of active coagulation factors and inhibition increases proportionally. The averages for the traditional methods were Quick PT (3.89 $\left.\mathrm{INR}_{\mathrm{Tot}}\right)$ and Owren PT (2.68 $\left.\mathrm{INR}_{\mathrm{Tot}}\right)$ and the difference 1.21 INR. After inhibition correction the averages were Quick PT (2.49 $\left.\mathrm{INR}_{\mathrm{Acf}}\right)$ and Owren PT $\left(2.30 \quad I_{N R}{ }_{A c f}\right)$ and the difference between Quick and Owren PT decreased to 0.19 INR.

More inactive coagulation factors (Multicalibrator) mean in our study lower patient INR; for Innovin the average was 2,85 INR and for Nycotest PT 2,44 INR, and using low-level inhibitory calibrators (Equalis) for Innovin 3,38 INR and for Nycotest PT 2,84 INR. 
Table 1. Four ISI Calibrator Kits Analyzed by the New-Generation PT Method Using Quick and Owren PT Reagents

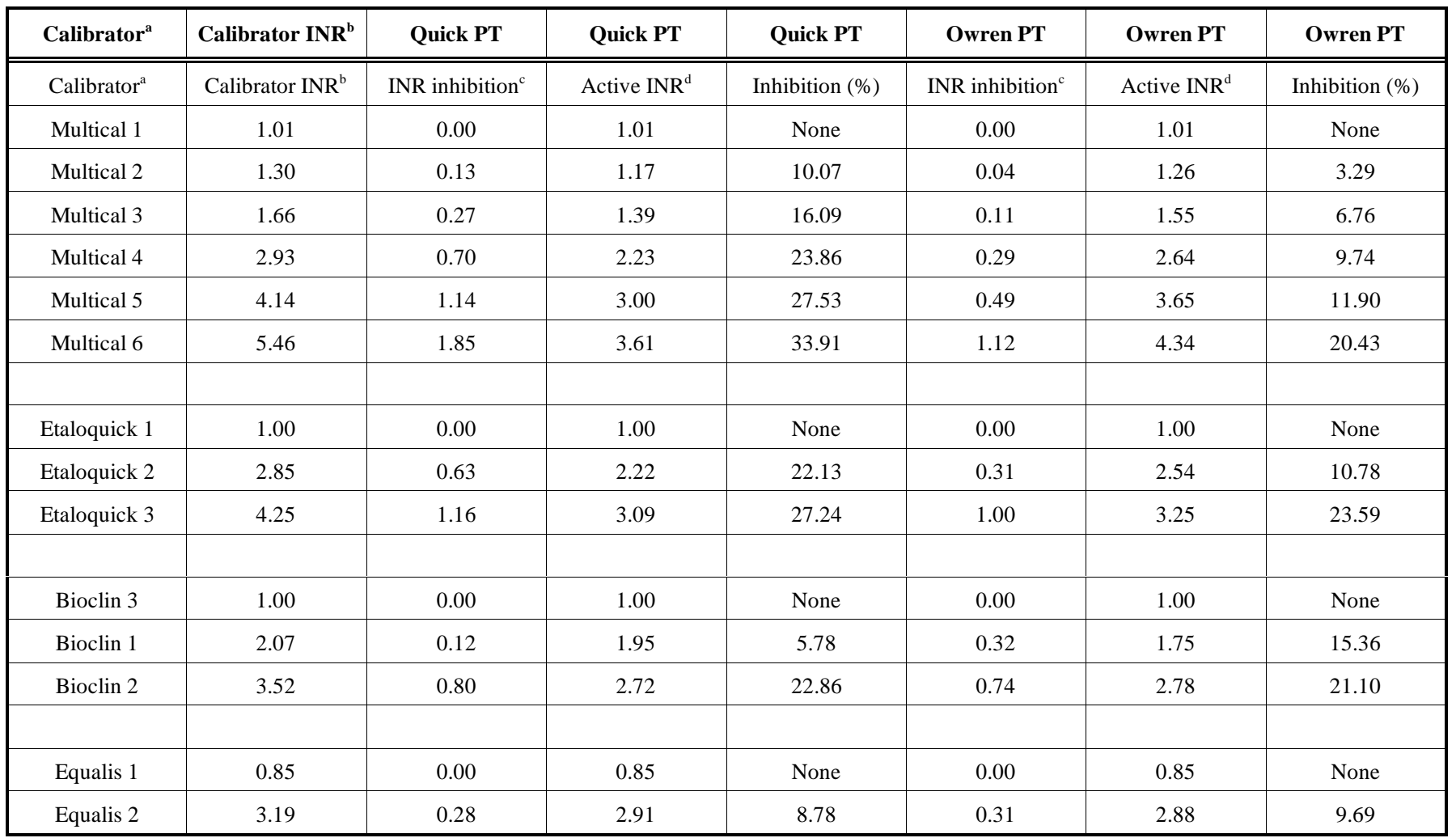

${ }^{\mathrm{a}}$ Arranged according to increasing INR values. ${ }^{\mathrm{b}}$ As given by the manufacturer. ${ }^{\mathrm{c}}$ Represents inhibition as INR. ${ }^{\mathrm{d}}$ Represents INR with no inhibitors present

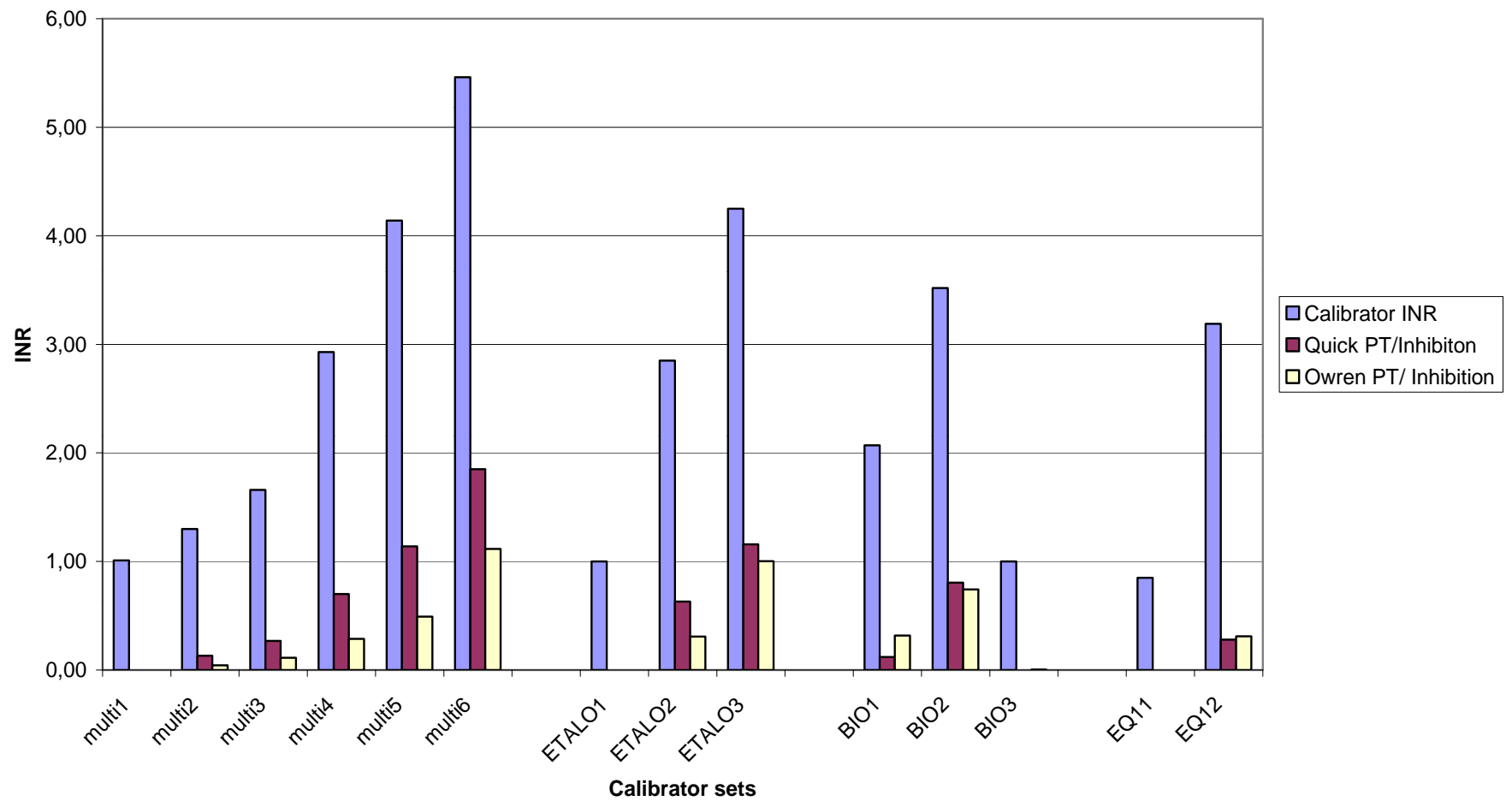

Fig. (1). Four ISI Calibrator Kits analyzed by the new-generation PT method using Quick and Owren PT reagents.

\section{DISCUSSION}

For a number of years new medications for anticoagulation therapy have been presented and anticipated without laboratory test control in an attempt to displace warfarin medication. The new medicines have proved inappropriate either in being too expensive or having serious side-effects. Since warfarin is a very cheap medicine it would be impor- 


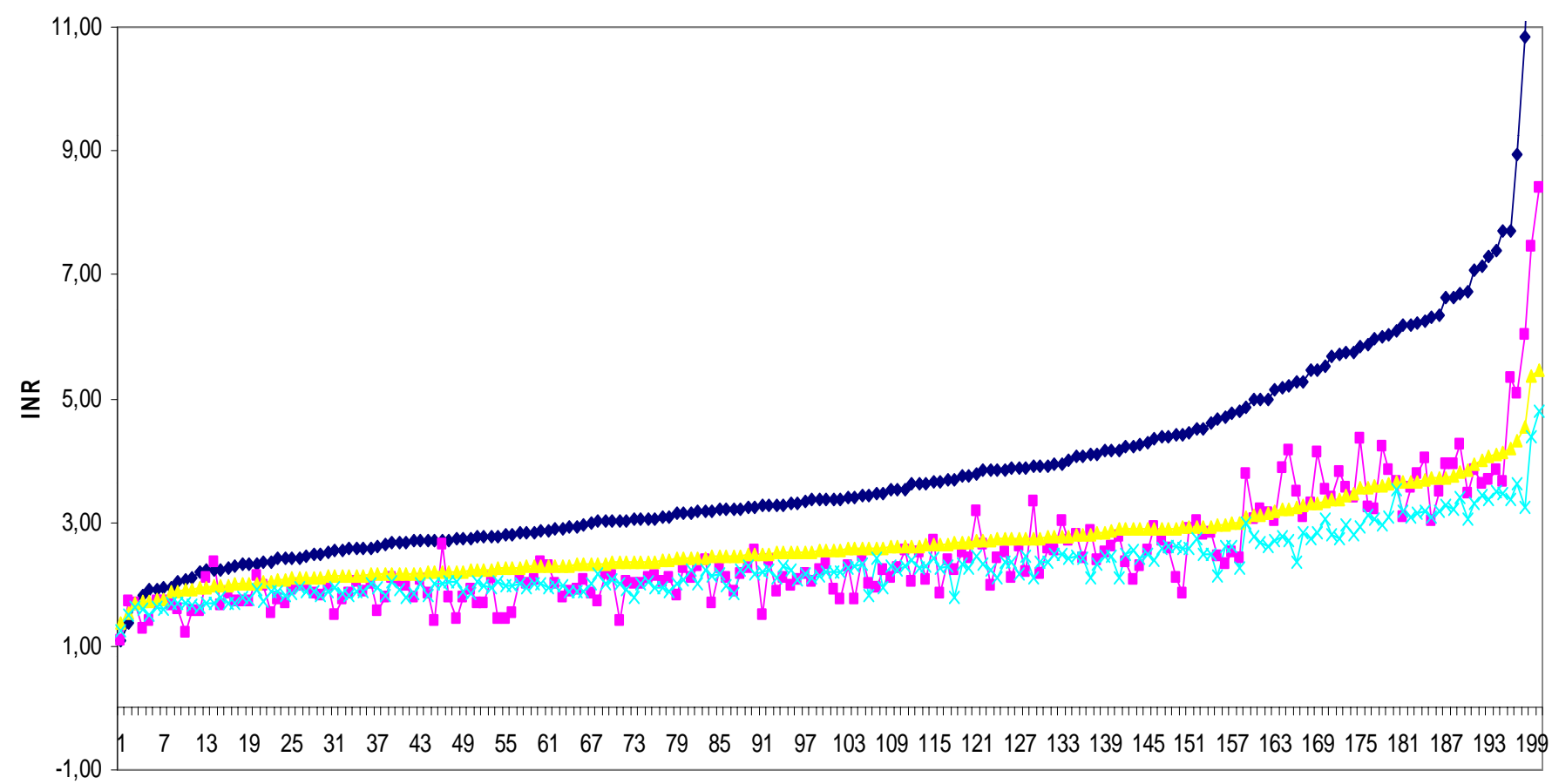

OAT patient samples from 1,08 to 14,26 INR $(n=200)$

$\multimap$ Quick PT INR, Tot $\multimap$ Quick PT, Active, INR $\multimap$ Owren PT INR, Tot $\nsim$ Owren PT, Active, INR

Fig. (2). Traditional $\mathrm{INR}_{\mathrm{Tot}}$ values determined by Quick and Owren PT and active coagulation factors (INR $\mathrm{Acf}_{\text {) }}$ for $200 \mathrm{OAT}$ patient plasmas in increasing order using the new-generation PT method and Etaloquick calibration.

tant to develop therapy with more attention to patient care in this competition.

The INR system transferred the responsibility to global laboratories to produce "the same INR from the same sample". This is a formidable challenge in view of different methods, reagents and instruments requiring harmonization. The current WHO calibration recommendation is complex [14] and calibration itself involves errors. We encountered this problem in our earlier study, where the same calibrator kit was used for seven different reagents [13].

In this present study we observed marked differences between calibrator kits in respect of inactive coagulation factors. The Quick PT is more sensitive to inhibition than the Owren and requires more calibrators and more correction at different INR levels. Different methods, thromboplastins and reagents have variable sensitivity to inactive coagulation factors and call for correction of inhibition or use of normal plasma. It is a cardinal error for calibration to contain inhibitory coagulation factors and hence the only possibility is to use normal-plasma dilution calibration.

An alternative means of determining the International Sensitivity Index is the use of freshly pooled plasmas from 20 normal individuals and 60 patients on OAT. These numbers of samples are necessary to obtain a precise calibration line for ISI calculation [19]. In this model the inhibition is constant or average at 2.5 INR. Current calibration involves average inhibition correction, which does not guarantee good INR result hamonization for individual patient sample.
The 200 OAT patient samples analysed show that the Quick PT needs more correction than the Owren to measure active coagulation factors $\left(\mathrm{INR}_{\mathrm{Acf}}\right)$. Inactive coagulation factors have more effect on Quick than on Owren PT, an observation which explains why using the same calibration for Quick PT reagents it is more difficult to harmonize INR than for Owren PT [12]. In our earlier study can we notice good harmony at INR 1 which get worse at higher INR values for seven different reagents [13]. This proves that the inhibition and disharmony increase together towards higher INR values. Inhibition correction for INR harmonizes the Quick and Owren PT methods very well through the measuring range. More accurate INR results mean better control for warfarin therapy and improved patient safety and possible less need for laboratory controls. The new method requires two measurements and a simple mathematical calculation for one patient sample. The current PT methods measure the sum of active coagulation factors and inhibition of inactive coagulation factors.

\section{CONCLUSION}

This new-generation PT method is applicable for the Quick and Owren PT methods. Measurement of active coagulation factors, $\mathrm{INR}_{\text {Acf }}(\mathrm{F}$ II, F VII, FX) provides a new possibility to develop anticoagulant therapy and more appropriate care for OAT patients. It helps to harmonize INR results for different PT methods and reagents. The new approach also provides an answer to the question posed in Clinical Chemistry: "Has the Time Arrived to Replace the Quick Prothrombin Time Test for Monitoring Oral Antico- 
agulant Therapy ?'[7]. Possibly this method can ensure the applicability of the Quick PT.

Normal plasma is suitable material for ISI calibration and is easily available and cheap material. Calibrators should not contain inhibitory coagulation factors if harmonization between PT reagents and methods is to be improved. The new method does not require need calibrators with inactive coagulation factors as does the current WHO- recommended calibration.

\section{LIST OF ABBREVIATIONS}

$\begin{array}{lll}\text { INR } & = & \text { International Normalized Ratio } \\ \text { ISI } & = & \text { International Sensitivity Index } \\ \text { OAT } & = & \text { Oral anticoagulation therapy } \\ \text { PIVKA } & = & \begin{array}{l}\text { Proteins Induced by Vitamin } \mathrm{K} \mathrm{Ab}- \\ \text { sence or Antagonist }\end{array} \\ \text { PT } & = & \text { Prothrombin time }\end{array}$

\section{COMPETING INTEREST}

The authors declare that they have no competing interests.

\section{AUTHOR'S CONTRIBUTIONS}

Juha Horsti was involved in planning and writing the manuscript, Helena Uppa in the experimental set-up and analysis of samples, and Juhani A. Vilpo in planning and writing the manuscript.

\section{REFERENCES}

[1] Odén A, Fahlén M. Oral anticoagulation and risk of death: a medical record linkage study. BMJ 2002; 325: 1073-5.

[2] Quick AJ, Stanley-Brown M, Bancroft FW. A study of the coagulation defect in hemophilia and in jaundice. Am J Med Sci 1935; 190: 501-11.

[3] Quick AJ. The prothrombin time in haemophilia and in obstructive jaundice. J Biol Chem 1935; 109: 73-4.

[4] Owren PA. Thrombotest. A new method for controlling anticoagulant therapy. Lancet 1959; 2: 754-8.
WHO Expert Committee on Biological Standardisation. Thirtythird Report. Technical Report Series 687. WHO Geneva, 1983; 81-105.

[6] International Committee for Standardisation in Haematology. International Committee on Thrombosis and Haemostastis. ICSH/ ICTH recommendations for reporting prothrombin time in oral anticoagulant control. Thromb Haemost 1985; 53: 155-6.

[7] Jackson CM, Esnouf MP. Has the Time Arrived to Replace the Quick Prothrombin Time Test for Monitoring Oral Anticoagulant Therapy. Clin Chem 2005; 51: 483-5.

[8] Cunningham MT, Johnson GF, Pennell BJ, Olson JD. The reliability of manufacturer-determined, instrument-specific international sensitivity index values for calculating the international normalised ratio. Am J Clin Path 1994; 102: 128-33.

[9] van Rijn JL, Schmidt NA, Rutten WP. Correction of instrumentand reagent-based differences in determination of the International Normalised Ratio (INR) for monitoring anticoagulant therapy. Clin Chem 1989; 35: 840-3.

[10] Ng VL, Levin J, Corash L, Gottfried EL. Failure of the International Normalised Ratio to generate consistent results within a local medical community. Am J Clin Path 1993; 99: 689-94.

[11] Horsti J. Agreement of Owren and Quick Prothrombin Times: Effects of Citrate and Calcium Concentrations and International Sensitivity Index Correction. Clin Chem 2001; 47: 940-4.

[12] Horsti J. Comparison of Quick and Owren Prothrombin Time with Regard to the Harmonisation of the International Normalised Ratio (INR) System. Clin Chem Lab Med 2002; 40: 399-403.

[13] Horsti J, Uppa H, Vilpo J. Poor agreement between different prothrombin time International Normalized Ratio (INR) methods: comparison of seven commercial reagents. Clin Chem 2005; 51: 553-60.

[14] Lindahl TL, Egberg N, Hillarp A, et al. INR calibration of Owrentype prothrombin time based on the relationship between PT\% and INR utilizing normal plasma samples. Thromb Haemost 2004; 91: 1223-31.

[15] Horsti J, Uppa H, Vilpo JA. A New Generation Prothrombin Time Method for INR. Med Chem J 2008; 2, 11-15.

[16] Stenflo J. Vitamin K, prothrombin and g-carboxyglutamatic acid. N Engl J Med 1977; 296: 624

[17] Hirsh J, Dalen JE, Anderson DR, et al. Oral Anticoagulants Mechanism of Action, Clinical Effectiveness, and Optimal Therapeutic Range. Chest 1998; 114: 445-69.

[18] Suttie JW. Synthesis of vitamin K-dependent proteins. FASEB J 1993; 7: 445-52.

[19] van den Besselaar AM, Witteveen E, Schaefer-van Mansfeld H, van Rijn C, van der Meer FJ. Effect of plasma pooling on the International Sensitivity Index of prothrombin time systems. Blood Coagul Fibrinol 1998; 9: 645-51. 